\title{
Binomial Fibonacci Power Sums
}

\author{
Kunle Adegoke \\ Department of Physics and Engineering Physics \\ Obafemi Awolowo University \\ 220005 Ile-Ife, Nigeria \\ adegoke00@gmail.com
}

2010 Mathematics Subject Classification: Primary 11B39; Secondary 11B37.

Keywords: Fibonacci number, Lucas number, summation identity, series, binomial coefficient.

\begin{abstract}
We evaluate various binomial sums involving the powers of Fibonacci and Lucas numbers.
\end{abstract}

\section{Introduction}

Our main goal in this paper is to evaluate the following sums of powers of Fibonacci and Lucas numbers involving the binomial coefficients:

$$
\begin{aligned}
& \sum_{k=0}^{n}( \pm 1)^{k}\left(\begin{array}{l}
n \\
k
\end{array}\right) F_{j(r k+s)}^{2 m}, \quad \sum_{k=0}^{n}( \pm 1)^{k}\left(\begin{array}{l}
n \\
k
\end{array}\right) L_{j(r k+s)}^{2 m}, \\
& \sum_{k=0}^{n}( \pm 1)^{k}\left(\begin{array}{l}
n \\
k
\end{array}\right) F_{j(2 r k+s)}^{2 m+1}, \quad \sum_{k=0}^{n}( \pm 1)^{k}\left(\begin{array}{l}
n \\
k
\end{array}\right) L_{j(2 r k+s)}^{2 m+1}
\end{aligned}
$$

thereby extending the work of Wessner [11], Hoggatt and Bicknell [3, 4], Long [6], Kiliç et al [7] and several previous researchers. Here $n$ is any non-negative integer, $j, m, r$ and $s$ are any integers and $F_{t}$ and $L_{t}$ are the Fibonacci and Lucas numbers.

There is a dearth of binomial cubic Fibonacci and Lucas identities. We will show that, for any non-negative integer $n$ and any integer $s$,

$$
\begin{gathered}
\sum_{k=0}^{n}\left(\begin{array}{l}
n \\
k
\end{array}\right) F_{k+s}^{3}=\frac{1}{5}\left(2^{n} F_{2 n+3 s}+3 F_{n-s}\right), \\
\sum_{k=0}^{n}\left(\begin{array}{l}
n \\
k
\end{array}\right) L_{k+s}^{3}=2^{n} L_{2 n+3 s}+3 L_{n-s},
\end{gathered}
$$




$$
\begin{gathered}
\sum_{k=0}^{n}\left(\begin{array}{l}
n \\
k
\end{array}\right)(-1)^{k} F_{k+s}^{3}=\frac{1}{5}\left((-1)^{n} 2^{n} F_{n+3 s}-(-1)^{s} 3 F_{2 n+s}\right), \\
\sum_{k=0}^{n}(-1)^{k}\left(\begin{array}{l}
n \\
k
\end{array}\right) L_{k+s}^{3}=(-1)^{n} 2^{n} L_{n+3 s}+(-1)^{s} 3 L_{2 n+s}, \\
\sum_{k=0}^{n}\left(\begin{array}{l}
n \\
k
\end{array}\right) 2^{k} F_{k+s}^{3}= \begin{cases}5^{n / 2-1}\left(F_{3 n+3 s}-(-1)^{s} 3 F_{s}\right), & n \text { even} ; \\
5^{(n-3) / 2}\left(L_{3 n+3 s}+(-1)^{s} 3 L_{s}\right) & n \text { odd },\end{cases} \\
\sum_{k=0}^{n}\left(\begin{array}{l}
n \\
k
\end{array}\right) 2^{k} L_{k+s}^{3}= \begin{cases}5^{n / 2}\left(L_{3 n+3 s}+(-1)^{s} 3 L_{s}\right), & n \text { even; } \\
5^{(n+1) / 2}\left(F_{3 n+3 s}-(-1)^{s} 3 F_{s}\right) & n \text { odd } .\end{cases}
\end{gathered}
$$

We will also derive the following binomial summation identities which we believe are new:

$$
\begin{gathered}
\sum_{k=0}^{n}(-1)^{k}\left(\begin{array}{l}
n \\
k
\end{array}\right) F_{2 j r+p}^{n-k} F_{p}^{k} F_{j(r k+s)}^{2}=\frac{1}{5}\left(F_{2 j r}^{n} L_{p n-2 j s}-(-1)^{j s} 2 F_{j r}^{n} L_{j r+p}^{n}\right), \\
\sum_{k=0}^{n}(-1)^{k}\left(\begin{array}{l}
n \\
k
\end{array}\right) F_{2 j r+p}^{n-k} F_{p}^{k} L_{j(r k+s)}^{2}=F_{2 j r}^{n} L_{p n-2 j s}+(-1)^{j s} 2 F_{j r}^{n} L_{j r+p}^{n}, \\
\sum_{k=0}^{n}(-1)^{k}\left(\begin{array}{l}
n \\
k
\end{array}\right) L_{2 j r+p}^{n-k} L_{p}^{k} F_{j(r k+s)}^{2}=\left\{\begin{array}{l}
5^{n / 2-1} F_{2 j r}^{n} L_{p n-2 j s}-(-1)^{j s} 5^{n-1} 2 F_{j r}^{n} F_{j r+p}^{n}, \quad n \text { even; } \\
5^{(n-1) / 2} F_{2 j r}^{n} F_{p n-2 j s}-(-1)^{j s} 5^{n-1} 2 F_{j r}^{n} F_{j r+p}^{n}, \quad n \text { odd },
\end{array}\right.
\end{gathered}
$$

and

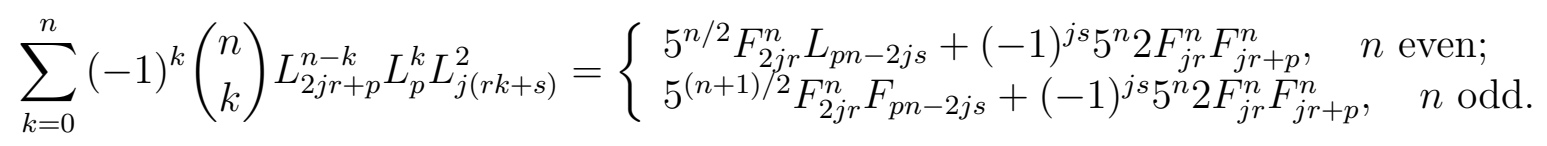

The Fibonacci numbers, $F_{n}$, and the Lucas numbers, $L_{n}$, are defined, for $n \in \mathbb{Z}$, through the recurrence relations

$$
F_{n}=F_{n-1}+F_{n-2},(n \geq 2), \quad F_{0}=0, F_{1}=1
$$

and

$$
L_{n}=L_{n-1}+L_{n-2},(n \geq 2), \quad L_{0}=2, L_{1}=1
$$

with

$$
F_{-n}=(-1)^{n-1} F_{n}, \quad L_{-n}=(-1)^{n} L_{n} .
$$

Throughout this paper, we denote the golden ratio, $(1+\sqrt{5}) / 2$, by $\alpha$ and write $\beta=(1-$ $\sqrt{5}) / 2=-1 / \alpha$, so that $\alpha \beta=-1$ and $\alpha+\beta=1$. 
Explicit formulas (Binet formulas) for the Fibonacci and Lucas numbers are

$$
F_{n}=\frac{\alpha^{n}-\beta^{n}}{\alpha-\beta}, \quad L_{n}=\alpha^{n}+\beta^{n}, \quad n \in \mathbb{Z} .
$$

Koshy [8] and Vajda [10] have written excellent books dealing with Fibonacci and Lucas numbers.

Our results emanate from the following general Fibonacci and Lucas summation identities (Lemma 2):

$$
\begin{gathered}
\sum_{k=0}^{n}\left(\begin{array}{l}
n \\
k
\end{array}\right) x^{n-k} z^{k} F_{j(r k+s)}^{m}=\frac{1}{(\sqrt{5})^{m}} \sum_{i=0}^{m}(-1)^{i(j s+1)}\left(\begin{array}{c}
m \\
i
\end{array}\right) \alpha^{(m-2 i) j s}\left(x+(-1)^{i j r} \alpha^{(m-2 i) j r} z\right)^{n}, \\
\sum_{k=0}^{n}\left(\begin{array}{l}
n \\
k
\end{array}\right) x^{n-k} z^{k} L_{j(r k+s)}^{m}=\sum_{i=0}^{m}(-1)^{i j s}\left(\begin{array}{c}
m \\
i
\end{array}\right) \alpha^{(m-2 i) j s}\left(x+(-1)^{i j r} \alpha^{(m-2 i) j r} z\right)^{n} .
\end{gathered}
$$

For lower $m$, the identities $(\mathrm{BF})$ and $(\mathrm{BL})$ are more useful in the equivalent form

$$
\begin{gathered}
\sum_{k=0}^{n}\left(\begin{array}{l}
n \\
k
\end{array}\right) x^{n-k} z^{k} F_{j(r k+s)}^{m}=\frac{1}{(\sqrt{5})^{m}} \sum_{i=0}^{m}(-1)^{i}\left(\begin{array}{c}
m \\
i
\end{array}\right) \beta^{i j s} \alpha^{(m-i) j s}\left(x+\beta^{i j r} \alpha^{(m-i) j r} z\right)^{n}, \\
\sum_{k=0}^{n}\left(\begin{array}{l}
n \\
k
\end{array}\right) x^{n-k} z^{k} L_{j(r k+s)}^{m}=\sum_{i=0}^{m}\left(\begin{array}{c}
m \\
i
\end{array}\right) \beta^{i j s} \alpha^{(m-i) j s}\left(x+\beta^{i j r} \alpha^{(m-i) j r} z\right)^{n} .
\end{gathered}
$$

When $m=1$, we have the weighted linear binomial identities:

$$
\begin{gathered}
\sum_{k=0}^{n}\left(\begin{array}{l}
n \\
k
\end{array}\right) x^{n-k} z^{k} F_{j(r k+s)}=\frac{1}{\sqrt{5}}\left(\alpha^{j s}\left(x+\alpha^{j r} z\right)^{n}-\beta^{j s}\left(x+\beta^{j r} z\right)^{n}\right), \\
\sum_{k=0}^{n}\left(\begin{array}{l}
n \\
k
\end{array}\right) x^{n-k} z^{k} L_{j(r k+s)}=\alpha^{j s}\left(x+\alpha^{j r} z\right)^{n}+\beta^{j s}\left(x+\beta^{j r} z\right)^{n},
\end{gathered}
$$

which are valid for $n$ a non-negative integer, $j, r, s$ integers and real or complex $x$ and $z$. Most linear binomial Fibonacci identities can be obtained from identities (F1) and (L1) by substituting appropriate choices of $n, j, r, s, x$ and $z$. For example, if we write $2 r$ for $r$ and set $x=(-1)^{j r}, z=1$ in (F1) and (L1), we obtain

$$
\begin{aligned}
& \sum_{k=0}^{n}(-1)^{j r k}\left(\begin{array}{l}
n \\
k
\end{array}\right) F_{j(2 r k+s)}=(-1)^{j r n} L_{j r}^{n} F_{j(r n+s)}, \\
& \sum_{k=0}^{n}(-1)^{j r k}\left(\begin{array}{l}
n \\
k
\end{array}\right) L_{j(2 r k+s)}=(-1)^{j r n} L_{j r}^{n} L_{j(r n+s)} ;
\end{aligned}
$$


which are valid for $n$ a non-negative integer and integers $r, s$ and $j$. The special case $(s=0)$ of identity (6) was also derived by Layman [9]. As another example of linear binomial Fibonacci identities that may be derived from (F1) and (L1), write $2 r$ for $r$ and set $x=(-1)^{j r}, z=-1$. This gives

$$
\begin{aligned}
& \sum_{k=0}^{n}(-1)^{(j r+1) k}\left(\begin{array}{l}
n \\
k
\end{array}\right) F_{j(2 r k+s)}= \begin{cases}5^{n / 2} F_{j r}^{n} F_{j(r n+s)}, & n \text { even; } \\
(-1)^{j r+1} 5^{(n-1) / 2} F_{j r}^{n} L_{j(r n+s)}, & n \text { odd } ;\end{cases} \\
& \sum_{k=0}^{n}(-1)^{(j r+1) k}\left(\begin{array}{l}
n \\
k
\end{array}\right) L_{j(2 r k+s)}= \begin{cases}5^{n / 2} F_{j r}^{n} L_{j(r n+s)}, & n \text { even; } \\
(-1)^{j r+1} 5^{(n+1) / 2} F_{j r}^{n} F_{j(r n+s)}, & n \text { odd } .\end{cases}
\end{aligned}
$$

Setting $\left(x=F_{p+j r}, z=-F_{p}\right)$ and also $\left(x=L_{p+j r}, z=-L_{p}\right)$ and making use of the identities of Hoggat et al, (see Lemma 3), where $p$ is any integer, we find

$$
\begin{aligned}
& \sum_{k=0}^{n}(-1)^{k}\left(\begin{array}{l}
n \\
k
\end{array}\right) F_{p+j r}^{n-k} F_{p}^{k} F_{j(r k+s)}=(-1)^{j s+1} F_{j r}^{n} F_{p n-j s} ; \\
& \sum_{k=0}^{n}(-1)^{k}\left(\begin{array}{l}
n \\
k
\end{array}\right) F_{p+j r}^{n-k} F_{p}^{k} L_{j(r k+s)}=(-1)^{j s+1} F_{j r}^{n} L_{p n-j s} ;
\end{aligned}
$$

and

$$
\begin{aligned}
\sum_{k=0}^{n}(-1)^{k}\left(\begin{array}{l}
n \\
k
\end{array}\right) L_{p+j r}^{n-k} L_{p}^{k} F_{j(r k+s)} & = \begin{cases}(-1)^{j s+1} 5^{\frac{n}{2}} F_{j r}^{n} F_{p n-j s}, & n \text { even } \\
(-1)^{j s+1} 5^{\frac{n-1}{2}} F_{j r}^{n} L_{p n-j s}, & n \text { odd }\end{cases} \\
\sum_{k=0}^{n}(-1)^{k}\left(\begin{array}{l}
n \\
k
\end{array}\right) L_{p+j r}^{n-k} L_{p}^{k} L_{j(r k+s)} & = \begin{cases}(-1)^{j s} 5^{\frac{n}{2}} F_{j r}^{n} L_{p n-j s}, & n \text { even; } \\
(-1)^{j s} 5^{\frac{n+1}{2}} F_{j r}^{n} F_{p n-j s}, & n \text { odd }\end{cases}
\end{aligned}
$$

Identities (9), (10) were obtained by Carlitz [1] while these and (11) and (12) may be found in Dresel [2]. The special case $(s=0)$ of (9) was also derived by Layman [9].

\section{Required identities and preliminary results}

Lemma 1. For real or complex $z$, let a given well-behaved function $h(z)$ have, in its domain, the representation $h(z)=\sum_{k=c_{1}}^{c_{2}} g(k) z^{f(k)}$ where $f(k)$ and $g(k)$ are given real sequences and $c_{1}, c_{2} \in[-\infty, \infty]$. Let $j$ be an integer. Then,

$$
\begin{gathered}
\sum_{k=c_{1}}^{c_{2}} g(k) z^{f(k)} F_{j f(k)}^{m}=\frac{1}{(\sqrt{5})^{m}} \sum_{i=0}^{m}(-1)^{i}\left(\begin{array}{c}
m \\
i
\end{array}\right) h\left(\beta^{i j} \alpha^{(m-i) j} z\right), \\
\sum_{k=c_{1}}^{c_{2}} g(k) z^{f(k)} L_{j f(k)}^{m}=\sum_{i=0}^{m}\left(\begin{array}{c}
m \\
i
\end{array}\right) h\left(\beta^{i j} \alpha^{(m-i) j} z\right) .
\end{gathered}
$$


Proof. We have

$$
\begin{aligned}
\sum_{k=c_{1}}^{c_{2}} g(k) z^{f(k)} F_{j f(k)}^{m} & =\sum_{k=c_{1}}^{c_{2}} g(k) z^{f(k)} \frac{\left(\alpha^{j f(k)}-\beta^{j f(k)}\right)^{m}}{(\sqrt{5})^{m}} \\
& =\frac{1}{(\sqrt{5})^{m}} \sum_{k=c_{1}}^{c_{2}} g(k) z^{f(k)} \sum_{i=0}^{m}(-1)^{i}\left(\begin{array}{c}
m \\
i
\end{array}\right) \beta^{i j f(k)} \alpha^{(m-i) j f(k)} \\
& =\frac{1}{(\sqrt{5})^{m}} \sum_{i=0}^{m}(-1)^{i}\left(\begin{array}{c}
m \\
i
\end{array}\right) \sum_{k=c_{1}}^{c_{2}} g(k)\left(\beta^{i j} \alpha^{(m-i) j} z\right)^{f(k)} \\
& =\frac{1}{(\sqrt{5})^{m}} \sum_{i=0}^{m}(-1)^{i}\left(\begin{array}{c}
m \\
i
\end{array}\right) h\left(\beta^{i j} \alpha^{(m-i) j} z\right) .
\end{aligned}
$$

The proof of $(\mathrm{L})$ is similar.

Since $\beta^{i} \alpha^{m-i}=(-1)^{i} \alpha^{m-2 i}$, identities $(\mathrm{F})$ and $(\mathrm{L})$ can also be written as

$$
\begin{gathered}
\sum_{k=c_{1}}^{c_{2}} g(k) z^{f(k)} F_{j f(k)}^{m}=\frac{1}{(\sqrt{5})^{m}} \sum_{i=0}^{m}(-1)^{i}\left(\begin{array}{c}
m \\
i
\end{array}\right) h\left((-1)^{i j} \alpha^{(m-2 i) j} z\right), \\
\sum_{k=c_{1}}^{c_{2}} g(k) z^{f(k)} L_{j f(k)}^{m}=\sum_{i=0}^{m}\left(\begin{array}{c}
m \\
i
\end{array}\right) h\left((-1)^{i j} \alpha^{(m-2 i) j} z\right) .
\end{gathered}
$$

Lemma 2. For non-negative integers $m$ and $n$, integers $j, r$ and $s$ and real or complex $x$ and $z$,

$$
\begin{gathered}
\sum_{k=0}^{n}\left(\begin{array}{l}
n \\
k
\end{array}\right) x^{n-k} z^{k} F_{j(r k+s)}^{m}=\frac{1}{(\sqrt{5})^{m}} \sum_{i=0}^{m}(-1)^{i(j s+1)}\left(\begin{array}{c}
m \\
i
\end{array}\right) \alpha^{(m-2 i) j s}\left(x+(-1)^{i j r} \alpha^{(m-2 i) j r} z\right)^{n} \\
\sum_{k=0}^{n}\left(\begin{array}{l}
n \\
k
\end{array}\right) x^{n-k} z^{k} L_{j(r k+s)}^{m}=\sum_{i=0}^{m}(-1)^{i j s}\left(\begin{array}{c}
m \\
i
\end{array}\right) \alpha^{(m-2 i) j s}\left(x+(-1)^{i j r} \alpha^{(m-2 i) j r} z\right)^{n}
\end{gathered}
$$

Proof. Consider the binomial identity

$$
h(z)=\sum_{k=0}^{n} g(k) z^{f(k)}=z^{s}\left(x+z^{r}\right)^{n},
$$

where

$$
f(k)=r k+s, \quad g(k)=\left(\begin{array}{l}
n \\
k
\end{array}\right) x^{n-k} .
$$


Thus,

$$
h\left((-1)^{i j} \alpha^{(m-2 i) j} z\right)=(-1)^{i j s} \alpha^{(m-2 i) j s} z^{s}\left(x+(-1)^{i j r} \alpha^{(m-2 i) j r} z^{r}\right)^{n} .
$$

Use of (14) and (15) in identity $\left(\mathrm{F}^{\prime}\right)$, with $c_{1}=0, c_{2}=n$ gives

$$
\sum_{k=0}^{n}\left(\begin{array}{l}
n \\
k
\end{array}\right) x^{n-k} z^{r k} F_{j(r k+s)}^{m}=\frac{1}{(\sqrt{5})^{m}} \sum_{i=0}^{m}(-1)^{i(j s+1)}\left(\begin{array}{c}
m \\
i
\end{array}\right) \alpha^{(m-2 i) j s}\left(x+(-1)^{i j r} \alpha^{(m-2 i) j r} z^{r}\right)^{n}
$$

from which identity (BF) follows when we write $z^{1 / r}$ for $z$. To prove (BL), use (14) and (15) in identity $\left(\mathrm{L}^{\prime}\right)$.

It is sometimes convenient to use the ( $\alpha$ vs $\beta$ ) version of identities (BF) and (BL):

$$
\begin{gathered}
\sum_{k=0}^{n}\left(\begin{array}{l}
n \\
k
\end{array}\right) x^{n-k} z^{k} F_{j(r k+s)}^{m}=\frac{1}{(\sqrt{5})^{m}} \sum_{i=0}^{m}(-1)^{i}\left(\begin{array}{c}
m \\
i
\end{array}\right) \beta^{i j s} \alpha^{(m-i) j s}\left(x+\beta^{i j r} \alpha^{(m-i) j r} z\right)^{n}, \\
\sum_{k=0}^{n}\left(\begin{array}{l}
n \\
k
\end{array}\right) x^{n-k} z^{k} L_{j(r k+s)}^{m}=\sum_{i=0}^{m}\left(\begin{array}{c}
m \\
i
\end{array}\right) \beta^{i j s} \alpha^{(m-i) j s}\left(x+\beta^{i j r} \alpha^{(m-i) j r} z\right)^{n} .
\end{gathered}
$$

Lemma 3 (Hoggatt et al [5]). For $p$ and $q$ integers,

$$
\begin{gathered}
L_{p+q}-L_{p} \alpha^{q}=-\beta^{p} F_{q} \sqrt{5}, \\
L_{p+q}-L_{p} \beta^{q}=\alpha^{p} F_{q} \sqrt{5}, \\
F_{p+q}-F_{p} \alpha^{q}=\beta^{p} F_{q}, \\
F_{p+q}-F_{p} \beta^{q}=\alpha^{p} F_{q} .
\end{gathered}
$$

Quadratic binomial Fibonacci identities may be obtained from $m=2$ in $\left(\mathrm{BF}^{\prime}\right)$ and $\left(\mathrm{BL}^{\prime}\right)$ :

$$
\begin{aligned}
& 5 \sum_{k=0}^{n}\left(\begin{array}{l}
n \\
k
\end{array}\right) x^{n-k} z^{k} F_{j(r k+s)}^{2}=\alpha^{2 j s}\left(x+\alpha^{2 j r} z\right)^{n}+\beta^{2 j s}\left(x+\beta^{2 j r} z\right)^{n} \\
& -2(-1)^{j s}\left(x+(-1)^{j r} z\right)^{n}, \\
& \sum_{k=0}^{n}\left(\begin{array}{l}
n \\
k
\end{array}\right) x^{n-k} z^{k} L_{j(r k+s)}^{2}=\alpha^{2 j s}\left(x+\alpha^{2 j r} z\right)^{n}+\beta^{2 j s}\left(x+\beta^{2 j r} z\right)^{n} \\
& +2(-1)^{j s}\left(x+(-1)^{j r} z\right)^{n} .
\end{aligned}
$$

Theorem 1. For non-negative integer $n$ and integers $j, r, s, p$,

$$
\sum_{k=0}^{n}(-1)^{k}\left(\begin{array}{l}
n \\
k
\end{array}\right) F_{2 j r+p}^{n-k} F_{p}^{k} F_{j(r k+s)}^{2}=\frac{1}{5}\left(F_{2 j r}^{n} L_{p n-2 j s}-(-1)^{j s} 2 F_{j r}^{n} L_{j r+p}^{n}\right), \quad p \neq 0,
$$




$$
\begin{gathered}
\sum_{k=0}^{n}(-1)^{k}\left(\begin{array}{l}
n \\
k
\end{array}\right) F_{2 j r+p}^{n-k} F_{p}^{k} L_{j(r k+s)}^{2}=F_{2 j r}^{n} L_{p n-2 j s}+(-1)^{j s} 2 F_{j r}^{n} L_{j r+p}^{n}, \quad p \neq 0, \\
\sum_{k=0}^{n}(-1)^{k}\left(\begin{array}{l}
n \\
k
\end{array}\right) L_{2 j r+p}^{n-k} L_{p}^{k} F_{j(r k+s)}^{2}=\left\{\begin{array}{l}
5^{n / 2-1} F_{2 j r}^{n} L_{p n-2 j s}-(-1)^{j s} 5^{n-1} 2 F_{j r}^{n} F_{j r+p}^{n}, \quad n \text { even } ; \\
5^{(n-1) / 2} F_{2 j r}^{n} F_{p n-2 j s}-(-1)^{j s} 5^{n-1} 2 F_{j r}^{n} F_{j r+p}^{n}, \quad n \text { odd },
\end{array}\right.
\end{gathered}
$$

and

$$
\sum_{k=0}^{n}(-1)^{k}\left(\begin{array}{l}
n \\
k
\end{array}\right) L_{2 j r+p}^{n-k} L_{p}^{k} L_{j(r k+s)}^{2}=\left\{\begin{array}{l}
5^{n / 2} F_{2 j r}^{n} L_{p n-2 j s}+(-1)^{j s} 5^{n} 2 F_{j r}^{n} F_{j r+p}^{n}, \quad n \text { even } ; \\
5^{(n+1) / 2} F_{2 j r}^{n} F_{p n-2 j s}+(-1)^{j s} 5^{n} 2 F_{j r}^{n} F_{j r+p}^{n}, \quad n \text { odd } .
\end{array}\right.
$$

Proof. Choose $x=F_{2 j r+p}, z=-F_{p}$ in (F2), noting Lemma 3, to obtain

$$
\begin{aligned}
5 \sum_{k=0}^{n}(-1)^{k}\left(\begin{array}{l}
n \\
k
\end{array}\right) F_{2 j r+p}^{n-k} F_{p}^{k} F_{j(r k+s)}^{2}= & F_{2 j r}^{n}\left(\alpha^{2 j s} \beta^{p n}+\alpha^{p n} \beta^{2 j s}\right) \\
& -2(-1)^{j s}\left(F_{2 j r+p}-(-1)^{j r} F_{p}\right)^{n},
\end{aligned}
$$

from which identity (20) follows. The same $(x, z)$ choice in (L2) produces identity (21).

Set $x=L_{2 j r+p}, z=-L_{p}$ in (F2), utilizing Lemma 3. This gives

$$
\begin{array}{r}
5 \sum_{k=0}^{n}(-1)^{k}\left(\begin{array}{l}
n \\
k
\end{array}\right) L_{2 j r+p}^{n-k} L_{p}^{k} F_{j(r k+s)}^{2}=F_{2 j r}^{n}(\sqrt{5})^{n}\left(\alpha^{p n-2 j s}+(-1)^{n} \beta^{p n-2 j s}\right) \\
-2(-1)^{j s}\left(L_{2 j r+p}-(-1)^{j r} L_{p}\right)^{n}
\end{array}
$$

and hence identity (22). The same $(x, z)$ choice in (L2) produces identity (23).

Cubic binomial Fibonacci identities may be obtained from $m=2$ in $\left(\mathrm{BF}^{\prime}\right)$ and $\left(\mathrm{BL}^{\prime}\right)$ :

$$
\begin{aligned}
5 \sqrt{5} \sum_{k=0}^{n}\left(\begin{array}{l}
n \\
k
\end{array}\right) x^{n-k} z^{k} F_{j(r k+s)}^{3}= & \alpha^{3 j s}\left(x+\alpha^{3 j r} z\right)^{n}-\beta^{3 j s}\left(x+\beta^{3 j r} z\right)^{n} \\
- & (-1)^{j s} 3 \alpha^{j s}\left(x+(-1)^{j r} \alpha^{j r} z\right)^{n} \\
& +(-1)^{j s} 3 \beta^{j s}\left(x+(-1)^{j r} \beta^{j r} z\right)^{n}, \\
\sum_{k=0}^{n}\left(\begin{array}{l}
n \\
k
\end{array}\right) x^{n-k} z^{k} L_{j(r k+s)}^{3}=\alpha^{3 j s}( & \left.+\alpha^{3 j r} z\right)^{n}+\beta^{3 j s}\left(x+\beta^{3 j r} z\right)^{n} \\
+ & (-1)^{j s} 3 \alpha^{j s}\left(x+(-1)^{j r} \alpha^{j r} z\right)^{n} \\
& +(-1)^{j s} 3 \beta^{j s}\left(x+(-1)^{j r} \beta^{j r} z\right)^{n} .
\end{aligned}
$$


Theorem 2. For non-negative integer $n$ and any integer $s$,

$$
\begin{gathered}
\sum_{k=0}^{n}\left(\begin{array}{l}
n \\
k
\end{array}\right) F_{k+s}^{3}=\frac{1}{5}\left(2^{n} F_{2 n+3 s}+3 F_{n-s}\right), \\
\sum_{k=0}^{n}\left(\begin{array}{l}
n \\
k
\end{array}\right) L_{k+s}^{3}=2^{n} L_{2 n+3 s}+3 L_{n-s}, \\
\sum_{k=0}^{n}\left(\begin{array}{l}
n \\
k
\end{array}\right)(-1)^{k} F_{k+s}^{3}=\frac{1}{5}\left((-1)^{n} 2^{n} F_{n+3 s}-(-1)^{s} 3 F_{2 n+s}\right), \\
\sum_{k=0}^{n}(-1)^{k}\left(\begin{array}{l}
n \\
k
\end{array}\right) L_{k+s}^{3}=(-1)^{n} 2^{n} L_{n+3 s}+(-1)^{s} 3 L_{2 n+s}, \\
\sum_{k=0}^{n}\left(\begin{array}{l}
n \\
k
\end{array}\right) 2^{k} F_{k+s}^{3}= \begin{cases}5^{n / 2-1}\left(F_{3 n+3 s}-(-1)^{s} 3 F_{s}\right), & n \text { even } ; \\
5^{(n-3) / 2}\left(L_{3 n+3 s}+(-1)^{s} 3 L_{s}\right) & n \text { odd },\end{cases} \\
\sum_{k=0}^{n}\left(\begin{array}{l}
n \\
k
\end{array}\right) 2^{k} L_{k+s}^{3}= \begin{cases}5^{n / 2}\left(L_{3 n+3 s}+(-1)^{s} 3 L_{s}\right), & n \text { even } ; \\
5^{(n+1) / 2}\left(F_{3 n+3 s}-(-1)^{s} 3 F_{s}\right) & n \text { odd } .\end{cases}
\end{gathered}
$$

Lemma 4. Let $a, b, c$ and $d$ be rational numbers and $\lambda$ an irrational number. Then,

$$
a+\lambda b=c+\lambda d \Longleftrightarrow a=c, \quad b=d
$$

Lemma 5. If $m$ is an integer and $(f(i))$ a real sequence, then,

$$
\begin{gathered}
\sum_{i=0}^{2 m} f(i)=f(m)+\sum_{i=0}^{m-1}(f(i)+f(2 m-i)), \\
\sum_{i=0}^{2 m+1} f(i)=f(2 m+1)-f(m)+\sum_{i=0}^{m}(f(i)+f(2 m-i)) .
\end{gathered}
$$

In particular, if $f(2 m-i)=f(i)$, then,

$$
\begin{gathered}
\sum_{i=0}^{2 m} f(i)=f(m)+2 \sum_{i=0}^{m-1} f(i), \\
\sum_{i=0}^{2 m+1} f(i)=f(2 m+1)-f(m)+2 \sum_{i=0}^{m} f(i) .
\end{gathered}
$$


Lemma 6. For $p$ and $q$ integers,

$$
\begin{gathered}
1+(-1)^{p} \alpha^{2 q}=\left\{\begin{array}{l}
(-1)^{p} \alpha^{q} F_{q} \sqrt{5}, \quad p \text { and } q \text { have different parity; } \\
(-1)^{p} \alpha^{q} L_{q}, \quad p \text { and } q \text { have the same parity. }
\end{array}\right. \\
1-(-1)^{p} \alpha^{2 q}=\left\{\begin{array}{l}
(-1)^{p-1} \alpha^{q} L_{q}, \quad p \text { and } q \text { have different parity; } \\
(-1)^{p-1} \alpha^{q} F_{q} \sqrt{5}, \quad p \text { and } q \text { have the same parity. }
\end{array}\right.
\end{gathered}
$$

Proof. We have

$$
\begin{aligned}
(-1)^{p+q}+(-1)^{p} \alpha^{2 q} & =\alpha^{p+q} \beta^{p+q}+\alpha^{p+2 q} \beta^{p} \\
& =\alpha^{p+q} \beta^{p}\left(\alpha^{q}+\beta^{q}\right) \\
& =(-1)^{p} \alpha^{q} L_{q}
\end{aligned}
$$

Similarly,

$$
(-1)^{p+q}-(-1)^{p} \alpha^{2 q}=(-1)^{p-1} \alpha^{q} F_{q} \sqrt{5} .
$$

Corresponding to (36) and (37) we have

$$
(-1)^{p+q}+(-1)^{p} \beta^{2 q}=(-1)^{p} \beta^{q} L_{q}
$$

and

$$
(-1)^{p+q}-(-1)^{p} \beta^{2 q}=(-1)^{p} \beta^{q} F_{q} \sqrt{5} .
$$

Identities (36), (37), (38) and (39) imply

$$
\begin{gathered}
(-1)^{q}+\alpha^{2 q}=\alpha^{q} L_{q}, \\
(-1)^{q}-\alpha^{2 q}=-\alpha^{q} F_{q} \sqrt{5} \\
(-1)^{q}+\beta^{2 q}=\beta^{q} L_{q} \\
(-1)^{q}-\beta^{2 q}=\beta^{q} F_{q} \sqrt{5}
\end{gathered}
$$

\section{Main results}

Theorem 3. Let $m$ and $n$ be non-negative integers and let $j, r$ and $s$ be any integers. Then,

$$
\begin{aligned}
& \sum_{k=0}^{n}\left(\begin{array}{l}
n \\
k
\end{array}\right) F_{j(r k+s)}^{2 m} \\
& = \begin{cases}5^{-m} \sum_{i=0}^{m-1}(-1)^{i(j s+j r n+1)}\left(\begin{array}{c}
2 m \\
i
\end{array}\right) L_{(m-i) j r}^{n} L_{(m-i)(j r n+2 j s)}+(-1)^{m(j s+1)}\left(\begin{array}{c}
2 m \\
m
\end{array}\right) 5^{-m} 2^{n}, \quad j m r \text { even } ; \\
5^{n / 2-m} \sum_{i=0}^{m-1}(-1)^{i(s+1)}\left(\begin{array}{c}
2 m \\
i
\end{array}\right) F_{(m-i) j r}^{n} L_{(m-i)(j r n+2 j s)}, & j m r \text { odd, } n \text { even } ; \\
5^{(n+1) / 2-m} \sum_{i=0}^{m-1}(-1)^{i s}\left(\begin{array}{c}
2 m \\
i
\end{array}\right) F_{(m-i) j r}^{n} F_{(m-i)(j r n+2 j s)}, & j m r \text { odd }, n \text { odd } .\end{cases}
\end{aligned}
$$




$$
\begin{aligned}
& \sum_{k=0}^{n}\left(\begin{array}{l}
n \\
k
\end{array}\right) L_{j(r k+s)}^{2 m} \\
& =\left\{\begin{array}{l}
\sum_{i=0}^{m-1}(-1)^{i(j s+j r n)}\left(\begin{array}{c}
2 m \\
i
\end{array}\right) L_{(m-i) j r}^{n} L_{(m-i)(j r n+2 j s)}+(-1)^{m j s}\left(\begin{array}{c}
2 m \\
m
\end{array}\right) 2^{n}, \quad j m r \text { even; } \\
5^{n / 2} \sum_{i=0}^{m-1}(-1)^{i s}\left(\begin{array}{c}
2 m \\
i
\end{array}\right) F_{(m-i) j r}^{n} L_{(m-i)(j r n+2 j s)}^{n}, \quad j m r \text { odd, } n \text { even; } \\
5^{(n+1) / 2} \sum_{i=0}^{m-1}(-1)^{i(s+1)}\left(\begin{array}{c}
2 m \\
i
\end{array}\right) F_{(m-i) j r}^{n} F_{(m-i)(j r n+2 j s)}, \quad j m r \text { odd, } n \text { odd. }
\end{array}\right.
\end{aligned}
$$

Proof. In (BF) write $2 m$ for $m$ and set $x=1$ and $z=1$. This gives

$$
5^{m} \sum_{k=0}^{n}\left(\begin{array}{l}
n \\
k
\end{array}\right) F_{j(r k+s)}^{2 m}=\sum_{i=0}^{2 m}(-1)^{i(j s+1)}\left(\begin{array}{c}
2 m \\
i
\end{array}\right) \alpha^{(m-i) 2 j s}\left(1+(-1)^{i j r} \alpha^{(m-i) 2 j r}\right)^{n} .
$$

Now, on account of Lemma 6, identity (34), we have

$$
1+(-1)^{i j r} \alpha^{(m-i) 2 j r}=\left\{\begin{array}{l}
(-1)^{i j r} \alpha^{(m-i) j r} L_{(m-i) j r}, \quad j r m \text { even; } \\
(-1)^{i} \alpha^{(m-i) j r} F_{(m-i) j r} \sqrt{5}, \quad j r m \text { odd }
\end{array}\right.
$$

Thus, using (47) in (46), we have

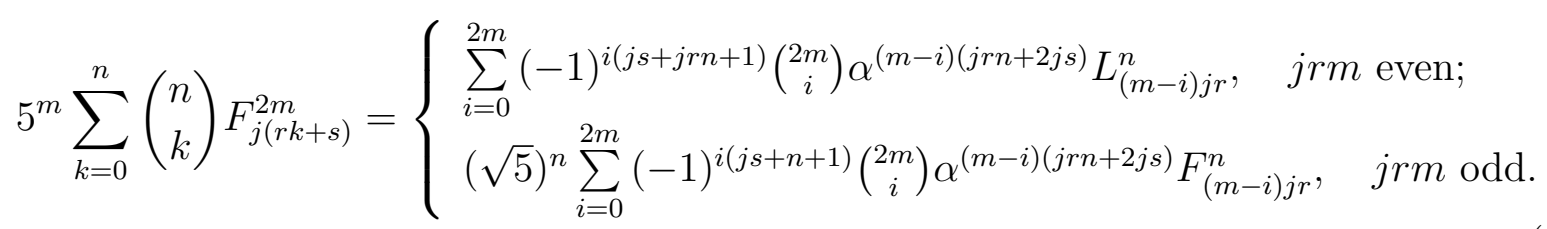

Observe that the left side of (48) evaluates to a rational number since it is the finite sum of rational numbers. Since,

$$
2 \alpha^{(m-i)(j r n+2 j s)}=L_{(m-i)(j r n+2 j s)}+F_{(m-i)(j r n+2 j s)} \sqrt{5}
$$

identity (44) now follows by comparing both sides of identity (48) in each case of $j m r$ even or $j m r$ odd, invoking Lemma 4 with $\lambda=\sqrt{5}$. Note the use of Lemma 5 , identity (30) to re-write the $(i=0$ to $2 m$ ) sum. The proof of identity (45) is similar; set $x=1$ and $z=1$ in (BL) and write $2 m$ for $m$.

Theorem 4. Let $m$ and $n$ be non-negative integers and let $j, r$ and $s$ be any integers. Then,

$$
\begin{aligned}
& \sum_{k=0}^{n}(-1)^{k}\left(\begin{array}{l}
n \\
k
\end{array}\right) F_{j(r k+s)}^{2 m} \\
& =\left\{\begin{array}{l}
5^{-m}(-1)^{n} \sum_{i=0}^{m-1}(-1)^{i(s+n+1)}\left(\begin{array}{c}
2 m \\
i
\end{array}\right) L_{(m-i) j r}^{n} L_{(m-i)(j r n+2 j s)}+(-1)^{s+1}\left(\begin{array}{c}
2 m \\
m
\end{array}\right) 5^{-m} 2^{n}, \quad j m r \text { odd } ; \\
5^{n / 2-m} \sum_{i=0}^{m-1}(-1)^{i(j s+1)}\left(\begin{array}{c}
2 m \\
i
\end{array}\right) F_{(m-i) j r}^{n} L_{(m-i)(j r n+2 j s)}, \quad j m r \text { even, } n \text { even } ; \\
-5^{(n+1) / 2-m} \sum_{i=0}^{m-1}(-1)^{i(j s+j r+1)}\left(\begin{array}{c}
2 m \\
i
\end{array}\right) F_{(m-i) j r}^{n} F_{(m-i)(j r n+2 j s)}, \quad j m r \text { even, } n \text { odd. }
\end{array}\right.
\end{aligned}
$$




$$
\begin{aligned}
& \sum_{k=0}^{n}(-1)^{k}\left(\begin{array}{l}
n \\
k
\end{array}\right) L_{j(r k+s)}^{2 m} \\
& =\left\{\begin{array}{l}
(-1)^{n} \sum_{i=0}^{m-1}(-1)^{i(s+n)}\left(\begin{array}{c}
2 m \\
i
\end{array}\right) L_{(m-i) j r}^{n} L_{(m-i)(j r n+2 j s)}+(-1)^{s}\left(\begin{array}{c}
2 m \\
m
\end{array}\right) 2^{n}, \quad j m r \text { odd; } \\
5^{n / 2} \sum_{i=0}^{m-1}(-1)^{i j s}\left(\begin{array}{c}
2 m \\
i
\end{array}\right) F_{(m-i) j r}^{n} L_{(m-i)(j r n+2 j s)}^{n}, \quad j m r \text { even, } n \text { even; } \\
-5^{(n+1) / 2} \sum_{i=0}^{m-1}(-1)^{i(j s+j r)}\left(\begin{array}{c}
2 m \\
i
\end{array}\right) F_{(m-i) j r}^{n} F_{(m-i)(j r n+2 j s)}, \quad j m r \text { even, } n \text { odd. }
\end{array}\right.
\end{aligned}
$$

Proof. In (BF) write $2 m$ for $m$ and set $x=1$ and $z=-1$. This gives

$$
5^{m} \sum_{k=0}^{n}(-1)^{k}\left(\begin{array}{l}
n \\
k
\end{array}\right) F_{j(r k+s)}^{2 m}=\sum_{i=0}^{2 m}(-1)^{i(j s+1)}\left(\begin{array}{c}
2 m \\
i
\end{array}\right) \alpha^{(m-i) 2 j s}\left(1-(-1)^{i j r} \alpha^{(m-i) 2 j r}\right)^{n} .
$$

Now, on account of Lemma 6, identity (35), we have

$$
1-(-1)^{i j r} \alpha^{(m-i) 2 j r}=\left\{\begin{array}{l}
(-1)^{i j r-1} \alpha^{(m-i) j r} F_{(m-i) j r} \sqrt{5}, \quad j r m \text { even; } \\
(-1)^{i-1} \alpha^{(m-i) j r} L_{(m-i) j r}, \quad j r m \text { odd }
\end{array}\right.
$$

Thus, using (53) in (52), we have

$5^{m} \sum_{k=0}^{n}(-1)^{k}\left(\begin{array}{l}n \\ k\end{array}\right) F_{j(r k+s)}^{2 m}=\left\{\begin{array}{l}\sum_{i=0}^{2 m}(-1)^{i n+i s+i-n}\left(\begin{array}{c}2 m \\ i\end{array}\right) \alpha^{(m-i)(j r n+2 j s)} L_{(m-i) j r}^{n}, \quad j r m \text { odd } ; \\ (\sqrt{5})^{n} \sum_{i=0}^{2 m}(-1)^{i j n r+i j s+i-n}\left(\begin{array}{c}2 m \\ i\end{array}\right) \alpha^{(m-i)(j r n+2 j s)} F_{(m-i) j r}^{n}, \quad j r m \text { even } .\end{array}\right.$

The left side of (54) evaluates to a rational number since it is the finite sum of rational numbers. Making use of identity (49), identity (50) follows by comparing both sides of identity (54) in each case of $j m r$ even or $j m r$ odd, invoking Lemma 4 with $\lambda=\sqrt{5}$. The proof of identity (51) is similar; put $x=1$ and $z=-1$ in (BL) and write $2 m$ for $m$.

The proofs of Theorems 5 and 6 are similar to those of Theorems 3 and 4 . We therefore omit the details and indicate only the appropriate choices of $x, z, m$ and $r$ to be made in identities $(\mathrm{BF})$ and $(\mathrm{BL})$ in each case.

Theorem 5. Let $m$ and $n$ be non-negative integers and let $j, r$ and $s$ be any integers. Then,

$$
\begin{aligned}
& \sum_{k=0}^{n}\left(\begin{array}{l}
n \\
k
\end{array}\right) F_{j(2 r k+s)}^{2 m+1} \\
& =\left\{\begin{array}{c}
5^{-m} \sum_{i=0}^{m}(-1)^{i(j s+1)}\left(\begin{array}{c}
2 m+1 \\
i
\end{array}\right) L_{(2 m+1-2 i) j r}^{n} F_{(2 m+1-2 i)(j r n+j s)}, \quad j r \text { even; } \\
5^{n / 2-m} \sum_{i=0}^{m}(-1)^{i(j s+1)}\left(\begin{array}{c}
2 m+1 \\
i
\end{array}\right) F_{(2 m+1-2 i) j r}^{n} F_{(2 m+1-2 i)(j r n+j s)}, \quad j r \text { odd }, n \text { even; } \\
5^{(n-1) / 2-m} \sum_{i=0}^{m}(-1)^{i(j s+1)}\left(\begin{array}{c}
2 m+1 \\
i
\end{array}\right) F_{(2 m+1-2 i) j r}^{n} L_{(2 m+1-2 i)(j r n+j s)}, \quad j r \text { odd }, n \text { odd } ;
\end{array}\right.
\end{aligned}
$$




$$
\begin{aligned}
& \sum_{k=0}^{n}\left(\begin{array}{l}
n \\
k
\end{array}\right) L_{j(2 r k+s)}^{2 m+1} \\
& =\left\{\begin{array}{l}
\sum_{i=0}^{m}(-1)^{i j s}\left(\begin{array}{c}
2 m+1 \\
i
\end{array}\right) L_{(2 m+1-2 i) j r}^{n} L_{(2 m+1-2 i)(j r n+j s)}, \quad j r \text { even } ; \\
5^{n / 2} \sum_{i=0}^{m}(-1)^{i j s}\left(\begin{array}{c}
2 m+1 \\
i
\end{array}\right) F_{(2 m+1-2 i) j r}^{n} L_{(2 m+1-2 i)(j r n+j s)}, \quad j r \text { odd, } n \text { even } ; \\
5^{(n+1) / 2} \sum_{i=0}^{m}(-1)^{i j s}\left(\begin{array}{c}
2 m+1 \\
i
\end{array}\right) F_{(2 m+1-2 i) j r}^{n} F_{(2 m+1-2 i)(j r n+j s)}, \quad j r \text { odd, } n \text { odd } .
\end{array}\right.
\end{aligned}
$$

Proof. Set $x=1, z=1$ and write $2 m+1$ for $m$ and $2 r$ for $r$ in identities (BF) and (BL). Note that

$$
1+\alpha^{(2 m+1-2 i) 2 j r}=\left\{\begin{array}{l}
\alpha^{(2 m+1-2 i) j r} L_{(2 m+1-2 i) j r}, j r \text { even; } \\
\alpha^{(2 m+1-2 i) j r} F_{(2 m+1-2 i) j r} \sqrt{5}, \quad j r \text { odd }
\end{array}\right.
$$

Theorem 6. Let $m$ and $n$ be non-negative integers and let $j, r$ and $s$ be any integers. Then,

$$
\begin{aligned}
& \sum_{k=0}^{n}(-1)^{k}\left(\begin{array}{l}
n \\
k
\end{array}\right) F_{j(2 r k+s)}^{2 m+1} \\
& = \begin{cases}(-1)^{n} 5^{-m} \sum_{i=0}^{m}(-1)^{i(j s+1)}\left(\begin{array}{c}
2 m+1 \\
i
\end{array}\right) L_{(2 m+1-2 i) j r}^{n} F_{(2 m+1-2 i)(j r n+j s)}, & j r \text { odd } ; \\
5^{n / 2-m} \sum_{i=0}^{m}(-1)^{i(j s+1)}\left(\begin{array}{c}
2 m+1 \\
i
\end{array}\right) F_{(2 m+1-2 i) j r}^{n} F_{(2 m+1-2 i)(j r n+j s)}, & j r \text { even, } n \text { even } ; \\
-5^{(n-1) / 2-m} \sum_{i=0}^{m}(-1)^{i(j s+1)}\left(\begin{array}{c}
2 m+1 \\
i
\end{array}\right) F_{(2 m+1-2 i) j r}^{n} L_{(2 m+1-2 i)(j r n+j s)}, & j r \text { even, } n \text { odd } ;\end{cases}
\end{aligned}
$$

$$
\begin{aligned}
& \sum_{k=0}^{n}(-1)^{k}\left(\begin{array}{l}
n \\
k
\end{array}\right) L_{j(2 r k+s)}^{2 m+1} \\
& =\left\{\begin{array}{c}
(-1)^{n} \sum_{i=0}^{m}(-1)^{i j s}\left(\begin{array}{c}
2 m+1 \\
i
\end{array}\right) L_{(2 m+1-2 i) j r}^{n} L_{(2 m+1-2 i)(j r n+j s)}, \quad j r \text { odd } ; \\
5^{n / 2} \sum_{i=0}^{m^{m}}(-1)^{i j s}\left(\begin{array}{c}
2 m+1 \\
i
\end{array}\right) F_{(2 m+1-2 i) j r}^{n} L_{(2 m+1-2 i)(j r n+j s)}, \quad j r \text { even, } n \text { even } ; \\
-5^{(n+1) / 2} \sum_{i=0}^{m}(-1)^{i j s}\left(\begin{array}{c}
2 m+1 \\
i
\end{array}\right) F_{(2 m+1-2 i) j r}^{n} F_{(2 m+1-2 i)(j r n+j s)}, \quad j r \text { even, } n \text { odd. }
\end{array}\right.
\end{aligned}
$$

Proof. Put $x=1, z=-1$ and write $2 m+1$ for $m$ and $2 r$ for $r$ in identities (BF) and (BL). Note that

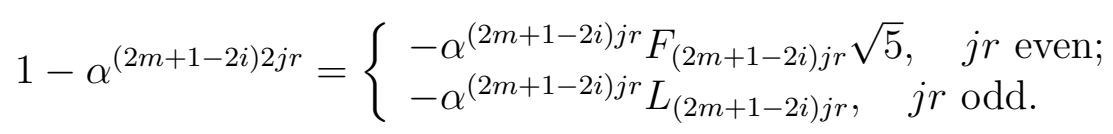




\section{References}

[1] L. Carlitz, Some classes of Fibonacci sums, The Fibonacci Quarterly 16:5 (1978), 411426.

[2] L. A. G. Dresel, Transformations of Fibonacci-Lucas identities, in Applications of Fibonacci Numbers, Vol. 5, Dordrecht: Kluwer, 1993, pp. 169-184.

[3] V. E. Hoggatt Jr and M. Bicknell, Some new Fibonacci identities, The Fibonacci Quarterly 2:1 (1964), 29-32.

[4] V. E. Hoggatt Jr and M. Bicknell, Fourth power identities from Pascal's triangle, The Fibonacci Quarterly 2:4 (1964), 261-266.

[5] V. E. Hoggatt, Jr., J. W. Phillips and H. T. Leonard, Jr., Twenty-four master identities, The Fibonacci Quarterly 9:1 (1971), 1-17.

[6] C. T. Long, Some binomial Fibonacci identities, in Applications of Fibonacci Numbers, Vol. 3, Dordrecht: Kluwer, 1990, pp. 241-254.

[7] E. Kiliç and I. Akkus and N. Ömür and Y. T. Ulutaş, Formulas for binomial sums including powers of Fibonacci and Lucas numbers, UPB Scientific Bulletin, Series A 77:4 (2015), 69-78.

[8] T. Koshy, Fibonacci and Lucas Numbers with Applications, Wiley-Interscience, 2001.

[9] J. W. Layman, Certain general binomial-Fibonacci sums, The Fibonacci Quarterly 15:3 (1977), 362-366.

[10] S. Vajda, Fibonacci and Lucas Numbers, and the Golden Section: Theory and Applications, Dover Press, 2008.

[11] J. Wessner, Binomial sums of Fibonacci powers, The Fibonacci Quarterly 4:4 (1966), 355-358.

Concerned with sequences: $\underline{\text { A000032, A000045. }}$. 